\title{
Approche par modélisation pluie-débit de la connaissance régionale de la ressource en eau : application au haut bassin du fleuve Sénégal
}

\section{Ansoumana Bodian}

\section{CpenEdition}

\section{Journals}

Édition électronique

URL : http://journals.openedition.org/cdg/1027

DOI : $10.4000 /$ cdg. 1027

ISSN : 2107-7266

Éditeur

UMR 245 - CESSMA

\section{Référence électronique}

Ansoumana Bodian, «Approche par modélisation pluie-débit de la connaissance régionale de la ressource en eau : application au haut bassin du fleuve Sénégal », Carnets de géographes [En ligne], 4 | 2012, mis en ligne le 01 septembre 2012, consulté le 24 septembre 2020. URL : http:// journals.openedition.org/cdg/1027 ; DOI : https://doi.org/10.4000/cdg.1027

Ce document a été généré automatiquement le 24 septembre 2020.

\section{(†) $\odot$

La revue Carnets de géographes est mise à disposition selon les termes de la Licence Creative Commons Attribution - Pas d'Utilisation Commerciale - Pas de Modification 4.0 International. 


\title{
Approche par modélisation pluie- débit de la connaissance régionale de la ressource en eau : application au haut bassin du fleuve Sénégal
}

\author{
Ansoumana Bodian
}

1 De nos jours la gestion des ressources en eau est un besoin qui s'exprime à tous les niveaux (local, régional, national, internationale) du fait des conséquences souvent dramatiques d'une pénurie en eau sur le plan humain, économique et politique. Cependant, l'on ne peut bien gérer une ressource que quand elle est connue. Malheureusement les dernières décennies ont vu une réduction considérable des capacités nationales, des pays qui se partagent le bassin du fleuve Sénégal, d'assurer le suivi hydrologique du fleuve et de ses affluents et de produire une information de qualité adaptée aux besoins des utilisateurs. Ainsi, la connaissance de la ressource en eau et de ses variations saisonnières au niveau du haut bassin de manière générale et dans la partie guinéenne du bassin en particulier présente des faiblesses à cause des séries de données hydrologiques tronquées ou manquantes. Les chroniques de débit sont souvent lacunaires, discontinues, de courte durée, et en conséquence sont difficilement exploitables pour une analyse hydrologique fiable.

Dans ce contexte, l'objectif principal de cette étude est de reconstituer les données hydrométriques surtout dans la partie guinéenne du bassin (ce pays vient de rejoindre l'OMVS en 2006, mais avec des données hydrométriques fragmentaires) en utilisant le modèle GR2M. Ceci permet de disposer de séries chronologiques de débits assez longues pour une meilleure estimation des ressources en eau et de leur fluctuation temporelle. À cette fin, on a procédé dans un premier temps à une caractérisation physiographique puis pluviométrique du haut bassin. Ensuite, une modélisation hydrologique a permis de réaliser le calage/validation des bassins grâce au modèle hydrologique GR2M sur une période de référence. En fin, nous avons évalué les impacts potentiels d'un changement climatique sur l'évolution des ressources en eau du haut bassin en utilisant 
les sorties de quatre modèles climatiques (CSMK3, HADCM3, MPEH5 et NCPCM) issues du dernier rapport de IPCC 2007 sous influence du scénario SRES A2.

La caractérisation du régime pluviométrique est basée sur une analyse statistique des pluies annuelles, mensuelles et journalières, ensuite une spatialisation des résultats des analyses statistiques a été effectuée. Ces cartes offrent un support de visualisation et de synthèse. Elles permettent de quitter la vision réduite à un point pour une vision globale de la pluie à l'échelle de la zone étudiée. Le calage/validation croisée du modèle a permis de choisir les paramètres qui traduisent le mieux la transformation de la pluie en débit. Une fois ces jeux de paramètres choisis pour chaque bassin, cette version du modèle a été appliquée sur l'ensemble de la série pour simuler les écoulements. Cette opération a permis d'étendre les séries de débits mensuels de trois bassins (Bafing Makana, Dakka Saidou et Sokotoro) de 1923 à 2005. Globalement, le modèle reproduit d'une manière satisfaisante la forme des hydrogrammes observés. Les débits de pointes calculés sont bien situés dans le temps même s'ils sont parfois sous-estimés (particulièrement pour le bassin de Bafing Makana pour la période allant de 1981 à 1985) ou surestimés. Les étiages par contre sont parfaitement reconstitués et on observe une bonne superposition des hydrogrammes des débits observés et calculés. En raison de la variété (taille, de physiographie, des états de surface et des caractéristiques des sols) des bassins, qui leur donne une sensibilité différente aux différents termes du climat, les impacts d'un potentiel changement climatique sont variables d'un bassin à l'autre. Ainsi, par rapport aux données observées, les scénarios utilisés prévoient sur nos bassins des variations d'écoulement moyen annuel qui dépendent étroitement des prévisions de précipitation de chaque modèle climatique. Une comparaison, intermodèles climatiques, des taux de variation des modules annuels aux différents horizons permet de constater que les modèles CSMK3, HADCM3 et MPEH5 présentent une similarité de prévisions pour les trois bassins. On assiste à une diminution progressive des écoulements de l'horizon 2030 à l'horizon 2090. Le modèle NCPCM se démarque des autres modèles climatiques et prévoit une augmentation progressive des écoulements de l'horizon 2030 à l'horizon 2090 sur l'ensemble des trois bassins. Ce dernier modèle est le plus optimiste quant à la prévision des écoulements futurs dans le haut bassin du fleuve Sénégal.

\author{
Discipline \\ Hydrologie \\ Directeur \\ Amadou Tahirou DIAW \\ Université \\ Université Cheikh Anta Diop de Dakar \\ Membres du jury de thèse, soutenue le 26 novembre 2011 \\ -Alioune KANE, Professeur, FLSH/UCAD, Président \\ -Raymond MALOU, Maître de Conférences, FST/UCAD, Rapporteur \\ -Luc DESCROIX, Directeur de Recherche, LTHE (Grenoble), Rapporteur \\ -Alain DEZETTER, Chargé de recherche, HSM (Montpellier), Examinateur \\ -Honoré DACOSTA, Chargé d'enseignement, FLSH/UCAD, Examinateur \\ -Amadou T. DIAW, Professeur, FLSH/UCAD, Directeur de thèse
}


Situation professionnelle actuelle

Chef de la Division hydrologie de la Direction de la Gestion et de la Planification

des Ressources en Eau du Sénégal

Courriel de l'auteur

dioung2001[at]yahoo.fr/bodianansoumana@gmail.com

INDEX

Thèmes : Carnets de soutenances 